\title{
Un critère d'indépendance pour une famille de représentations $\ell$-adiques
}

Jean-Pierre Serre

Résumé. We give a criterion for the independence of a family of $\ell$-adic Galois representations of a number field.

Classification mathématique par sujets (2010). 14 F.

Mots-clefs. Galois representations, cohomology, independence.

\section{Introduction}

Soit $k$ un corps de nombres, de clôture algébrique $\bar{k}$, et soit $A$ une variété abélienne sur $k$, de dimension $d$. Comme on sait, de telles données définissent, pour tout nombre premier $\ell$, une représentation $\ell$-adique

$$
\rho_{\ell}: \Gamma_{k} \rightarrow \operatorname{Aut}\left(T_{\ell}(A)\right) \cong \mathbf{G L}_{2 d}\left(\mathbf{Z}_{\ell}\right),
$$

où $\Gamma_{k}=\operatorname{Gal}(\bar{k} / k)$, et $T_{\ell}(A)$ est le $\ell$-ième module de Tate de $A$ sur $\bar{k}$. La famille des $\rho_{\ell}$ s'identifie à un homomorphisme continu

$$
\rho: \Gamma_{k} \rightarrow \prod_{\ell} \operatorname{Aut}\left(T_{\ell}(A)\right) \cong \prod_{\ell} \mathbf{G L}_{2 d}\left(\mathbf{Z}_{\ell}\right) .
$$

Lorsqu'on s'intéresse au sous-groupe $\rho\left(\Gamma_{k}\right)$ de $\prod_{\ell} \mathbf{G L}_{2 d}\left(\mathbf{Z}_{\ell}\right)$, il est commode de savoir que $\rho\left(\Gamma_{k}\right)$ est le produit direct des $\rho_{\ell}\left(\Gamma_{k}\right)$, autrement dit, que les $\rho_{\ell}$ sont "indépendants". Bien entendu ce n'est pas toujours vrai, mais on peut démontrer (cf. [Se 86]) que cela le devient après une extension finie convenable de $k$; autrement dit, les $\rho_{\ell}$ sont "presque indépendants".

Je me propose de reprendre cette question en mettant en évidence les propriétés des $\rho_{\ell}$ qui entraînent la presque indépendance. Comme on le verra au $\S 2$, ce sont des propriétés de ramification, analogues à ce que l'on appelle la "semi-stabilité" ; curieusement, les éléments de Frobenius, si utiles en d'autres circonstances, ne jouent ici aucun rôle.

L'intérêt de cette axiomatisation est qu'on peut l'appliquer à des situations plus générales que celle des variétés abéliennes, par exemple à la cohomologie $\ell$-adique des variétés algébriques sur un corps de nombres, cf. \$3.2. Un résultat très voisin 
avait d'ailleurs été obtenu il y a une quinzaine d'années par M. J. Larsen et R. Pink dans des lettres (datées du 23/5/95 et 26/5/95) dont le contenu n'a malheureusement pas été publié jusqu'à présent.

La démonstration du théorème principal (théorème 1 du §2) est donnée au §8. Elle repose sur diverses propriétés des corps de nombres et des groupes linéaires (corps de classes, théorème de Hermite-Minkowski, théorèmes de Jordan et de Nori); ces propriétés font l'objet des §§4-7.

Remerciement. Cet article doit beaucoup à L. Illusie : il m'a encouragé à l'écrire, il m'a fourni de nombreuses références et il m'a communiqué ([Il 10]) une démonstration d'un résultat auxiliaire essentiel, qui avait été démontré auparavant, sous une forme un peu différente, par N. Katz et G. Laumon. Je lui en suis très reconnaissant.

\section{§1. La notion d'indépendance}

Soit $\Gamma$ un groupe, et soit $\rho_{i}: \Gamma \rightarrow G_{i}$ une famille d'homomorphismes de $\Gamma$ dans des groupes $G_{i}$ indexés par un ensemble $I$. Cela revient à se donner un homomorphisme

$$
\rho=\left(\rho_{i}\right): \Gamma \rightarrow \prod_{i \in I} G_{i} .
$$

On dit que les $\rho_{i}$ sont indépendants si la propriété suivante est satisfaite :

(R) $\rho(\Gamma)=\prod \rho_{i}(\Gamma)$.

Autrement dit, si $\gamma_{i}$ est une famille quelconque d'éléments de $\Gamma$, il existe $\gamma \in \Gamma$ tel que $\rho_{i}(\gamma)=\rho_{i}\left(\gamma_{i}\right)$ pour tout $i$.

Il y a une propriété plus faible que l'on peut considérer :

(RO) $\rho(\Gamma)$ est un sous-groupe d'indice fini de $\prod \rho_{i}(\Gamma)$.

A partir de maintenant, on suppose que $\Gamma$ est un groupe profini, que les $G_{i}$ sont localement compacts, et que les $\rho_{i}$ sont continus (de sorte que les $\rho_{i}(\Gamma)$ sont des groupes profinis). On s'intéresse à la propriété :

(PR) Il existe un sous-groupe ouvert $\Gamma^{\prime}$ de $\Gamma$ tel que les restrictions des $\rho_{i}$ à $\Gamma^{\prime}$ vérifient $(\mathrm{R})$. [Noter que $\Gamma^{\prime}$ est d'indice fini dans $\Gamma$, puisque $\Gamma$ est compact.]

On dit alors que les $\rho_{i}$ sont presque indépendants.

On a $(\mathrm{R}) \Rightarrow(\mathrm{RO}) \Rightarrow(\mathrm{PR})$ : c'est clair pour $(\mathrm{R}) \Rightarrow(\mathrm{RO})$, et ce n'est pas difficile pour $(\mathrm{RO}) \Rightarrow(\mathrm{PR})$.

Remarque. On peut aussi exprimer (R) comme une propriété des noyaux $N_{i}$ des $\rho_{i}$. Si l'on pose $N_{i}^{\prime}=\bigcap_{j \neq i} N_{j}$, la condition (R) est équivalente à chacune des deux conditions suivantes :

(R1) $\Gamma=N_{i} . N_{i}^{\prime}$ pour tout $i$.

(R2) $\Gamma$ est engendré (topologiquement) par les $N_{i}^{\prime}$. 
Vol. 88 (2013) Un critère d'indépendance pour une famille de représentations $\ell$-adiques 543

[Lorsque $I$ est fini, cela se démontre par récurrence sur le nombre d'éléments de $I$; le cas général s'en déduit par passage à la limite, en utilisant la compacité de $\Gamma$.]

On peut préciser (R2) : si l'on note $\Gamma^{\prime}$ le plus petit sous-groupe fermé de $\Gamma$ contenant les $N_{i}^{\prime}$, alors $\Gamma^{\prime}$ est le plus grand sous-groupe fermé de $\Gamma$ sur lequel les $\rho_{i}$ sont indépendants.

\section{§2. Enoncé du théorème}

Il y a trois données :

a) $k$ est un corps de nombres de clôture algébrique $\bar{k}$; on note $\Gamma_{k}$ le groupe de Galois $\operatorname{Gal}(\bar{k} / k)$.

b) $L$ est un ensemble de nombres premiers.

c) Pour tout $\ell \in L, G_{\ell}$ est un groupe de Lie $\ell$-adique localement compact ${ }^{1}$, et $\rho_{\ell}: \Gamma_{k} \rightarrow G_{\ell}$ est un homomorphisme continu.

On fait deux sortes d'hypothèses :

2.1. On suppose que la famille des $\rho_{\ell}\left(\Gamma_{k}\right)$ est bornée, i.e. qu'elle satisfait à la condition suivante :

(B) Il existe un entier $n \geqslant 0$ tel que, pour tout $\ell \in L, \rho_{\ell}\left(\Gamma_{k}\right)$ soit isomorphe à un sous-quotient de $\mathbf{G L}_{n}\left(\mathbf{Z}_{\ell}\right)$.

[Rappelons qu'un "sous-quotient" d'un groupe $A$ est un quotient d'un sous-groupe de $A$. Bien sûr, il s'agit ici de sous-groupes fermés.]

Les cas particuliers les plus intéressants sont ceux où l'on a $G_{\ell}=\mathbf{G L}_{n_{\ell}}\left(\mathbf{Z}_{\ell}\right)$, ou $G_{\ell}=\mathbf{G L}_{n_{\ell}}\left(\mathbf{F}_{\ell}\right)$, avec des $n_{\ell}$ bornés (par exemple constants).

2.2. On fait une hypothèse du genre "semi-stabilité" sur la famille des $\rho_{\ell}$. Pour l'énoncer, notons $V_{k}$ l'ensemble des places non archimédiennes de $k$. Si $v \in V_{k}$, notons $k_{v}$ le complété de $k$ en $v$, notons $p_{v}$ la caractéristique résiduelle de $v$ et choisissons un prolongement $\bar{v}$ de $v$ à $\bar{k}$. Notons $I_{\bar{v}}$ le groupe d'inertie correspondant à $\bar{v}$; c'est un sous-groupe fermé de $\Gamma_{k}$; à conjugaison près, il ne dépend que de $v$.

Avec ces notations, l'hypothèse dont on a besoin s'énonce de la manière suivante: (ST) Il existe un sous-ensemble fini $S$ de $V_{k}$ tel que :

(ST1) Si $v \notin S$ et $\ell \neq p_{v}$, alors $\rho_{\ell}\left(I_{\bar{v}}\right)=1$, i.e. $\rho_{\ell}$ est non ramifié en $v$.

(ST2) Si $v \in S$ et $\ell \neq p_{v}$, alors $\rho_{\ell}\left(I_{\bar{v}}\right)$ est un pro- $\ell$-groupe.

[Noter que l'on ne fait aucune hypothèse sur les $\rho_{\ell}\left(I_{\bar{v}}\right)$ lorsque $\ell=p_{v}$.]

Lorsque $G_{\ell}=\mathbf{G L}_{n}\left(\mathbf{Z}_{\ell}\right)$, la condition (ST2) est moins restrictive que la condition habituelle de semi-stabilité, où l'on exige que $\rho_{\ell}\left(I_{\bar{v}}\right)$ soit formé d'éléments unipotents.

Il est commode d'introduire une notion analogue à la potentielle semi-stabilité :

\footnotetext{
${ }^{1}$ On ne perdrait rien si l'on supposait que les $G_{\ell}$ sont compacts, vu que l'on peut supposer que les $\rho_{\ell}$ sont surjectifs.
} 
(PST) Il existe une extension finie de $k$ pour laquelle (ST) est satisfaite. [Plus explicitement : il existe une sous-extension finie $k_{1}$ de $\bar{k}$ telle que la famille des $\rho_{\ell} \mid \Gamma_{k_{1}}$ satisfasse à (ST).]

Noter que, dès que (ST) est satisfaite pour une extension $k_{1}$ de $k$, elle l'est aussi pour toute extension finie de $k$ contenant $k_{1}$.

2.3. Le théorème que nous avons en vue dit que les propriétés (B) et (PST) entraînent la propriété (PR) du $\S 1$. Autrement dit :

Théorème 1. Si la famille des $\rho_{\ell}\left(\Gamma_{k}\right)$ est bornée au sens de (B), et si la condition (PST) est satisfaite, il existe une extension finie de $k$ sur laquelle les $\rho_{\ell}$ sont indépendants.

On peut reformuler cet énoncé en termes d'extensions de $k$ : notons $N_{\ell}$ le noyau de $\rho_{\ell}$ et $k_{\ell}$ le sous-corps de $\bar{k}$ fixé par $N_{l}$; posons $N_{\ell}^{\prime}=\bigcap_{\ell^{\prime} \neq \ell} N_{\ell^{\prime}}$ et notons $k_{\ell}^{\prime}$ le corps fixé par $N_{\ell}^{\prime}$, autrement dit le corps engendré par les $k_{\ell^{\prime}}$ avec $\ell^{\prime} \neq \ell$. Le corps $k^{\text {ind }}=\bigcap k_{\ell}^{\prime}$ correspond, par la théorie de Galois, au plus petit sous-groupe fermé de $\Gamma_{k}$ contenant les $N_{\ell}^{\prime}$. Avec ces notations, le théorème 1 est équivalent à :

Théorème $\mathbf{1}^{\prime}$. Si les conditions $(\mathrm{B})$ et $(\mathrm{PST})$ sont satisfaites, le corps $k^{\mathrm{ind}}=\bigcap_{\ell} k_{\ell}^{\prime}$ défini ci-dessus est une extension finie de $k$.

De plus, $k^{\text {ind }}$ est la plus petite extension de $k$ sur laquelle les $\rho_{\ell}$ sont indépendants ; on peut l'appeler le "corps d'indépendance" des $\rho_{\ell}$.

La démonstration des théorèmes 1 et $1^{\prime}$ sera donnée au $\S 8$.

\section{§3. Exemples et contre-exemples}

Dans chacun des exemples ci-dessous, l'ensemble $L$ est l'ensemble de tous les nombres premiers, et $G_{\ell}$ est isomorphe à $\mathbf{G L}_{n}\left(\mathbf{Q}_{\ell}\right)$, avec $n$ fixe. Cette dernière hypothèse entraîne que le groupe $\rho_{\ell}\left(\Gamma_{k}\right)$ est isomorphe à un sous-groupe fermé de $\mathbf{G L}_{n}\left(\mathbf{Z}_{\ell}\right)$, de sorte que la condition (B) est satisfaite.

3.1. Variétés abéliennes et quasi-abéliennes. Si $A$ est une variété abélienne de dimension $d$ sur $k$, les modules de Tate $T_{\ell}(A)$ fournissent des représentations $\ell$-adiques de dimension $2 d$ de $\Gamma_{k}$ qui satisfont à (PST) en vertu du théorème de Grothendieck et Mumford sur la semi-stabilité des modèles de Néron ([SGA 7 I], exposé IX, voir aussi [BLR 90], §7.4).

D'après le théorème 1 , ces représentations sont presque indépendantes : on retrouve ainsi un résultat démontré un peu différemment dans [Se 86]. Noter qu'ici les corps $k_{\ell}$ ont une interprétation simple $: k_{\ell}$ est le corps de rationalité des points de $A(\bar{k})$ d'ordre une puissance de $\ell$, et $k_{\ell}^{\prime}$ est le corps de rationalité des points de $A(\bar{k})$ d'ordre fini premier à $\ell$. 
Ces résultats s'appliquent aussi au cas des schémas en groupes quasi-abéliens; ce cas a été utilisé par Hrushovski, cf. [Bo 00].

3.2. Cohomologie $\ell$-adique. Plus généralement, si $X$ est un schéma séparé de type fini sur $k$, la condition (PST) est satisfaite par les représentations $\ell$-adiques associées aux groupes de cohomologie à support propre $H_{c}^{i}\left(\bar{X}, \mathbf{Q}_{\ell}\right)$, ainsi que par les groupes de cohomologie $H^{i}\left(\bar{X}, \mathbf{Q}_{\ell}\right)$ à support quelconque. En effet :

a) La condition (ST1) est satisfaite d'après les théorèmes d'existence de "stratifications" dus à N. Katz et G. Laumon [KL 86], th. 3.1.2 et th. 3.3.2.

b) Si $S$ est choisi comme dans (ST1), il résulte d'un théorème de Berthelot [Be 96], prop. 6.3.2, que, pour tout $v \in S$, il existe un sous-groupe ouvert normal $U_{\bar{v}}$ de $I_{\bar{v}}$ qui opère de façon unipotente sur les $H_{c}^{i}\left(\bar{X}, \mathbf{Q}_{\ell}\right)$ et les $H^{i}\left(\bar{X}, \mathbf{Q}_{\ell}\right)$, pourvu que $\ell \neq p_{v}$. [La démonstration de Berthelot est basée sur la théorie des altérations de de Jong, cf. [Jo 96].] Choisissons une extension galoisienne $k_{v}^{\prime}$ du corps local $k_{v}$ telle que $\Gamma_{k_{v}^{\prime}} \cap I_{\bar{v}} \subset U_{\bar{v}}$. Un argument d'approximation bien connu montre qu'il existe une extension galoisienne finie $k_{1}$ de $k$ dont les complétés locaux aux places au-dessus de $S$ contiennent les $k_{v}^{\prime}$. On a alors $\Gamma_{k_{1}} \cap I_{\bar{v}} \subset U_{\bar{v}}$ pour tout $v \in S$, ce qui montre que la condition (ST2) est satisfaite sur $k_{1}$.

Problème (cf. [Se 91], 10.1 ?). Au lieu de supposer, comme nous venons de le faire, que $k$ est un corps de nombres, supposons seulement que $k$ est une extension de type fini de $\mathbf{Q}$. Comme ci-dessus, soit $X$ un schéma séparé de type fini sur $k$. Est-il encore vrai que les représentations $\ell$-adiques de $\Gamma_{k}$ fournies par les $H_{c}^{i}\left(\bar{X}, \mathbf{Q}_{\ell}\right)$ et les $H^{i}\left(\bar{X}, \mathbf{Q}_{\ell}\right)$ sont presque indépendantes ? ${ }^{2}$

3.3. Mariage "carpe-lapin". On peut partir de deux familles de $\rho_{\ell}$ satisfaisant aux hypothèses (B) et (PST), et pour chaque $\ell$ choisir au hasard l'un des deux $\rho_{\ell}$; on obtient encore une famille presque indépendante. Exemple $:$ pour $\ell \equiv 1(\bmod 4)$ prendre la représentation $\ell$-adique associée à la fonction de Ramanujan, et pour les autres $\ell$ la représentation $\ell$-adique associée à la courbe elliptique d'équation $y^{2}-y=x^{3}-x^{2}$.

\subsection{Exemple montrant que la condition (PST) ne peut pas être entièrement} supprimée. Soit $k=\mathbf{Q}$. Choisissons un nombre premier $p>2$, ainsi qu'une suite infinie $\ell_{1}<\ell_{2}<\cdots$ de nombres premiers tels que $\ell_{i} \equiv 1\left(\bmod p^{i}\right)$. Soit $L=$ $\left\{\ell_{1}, \ell_{2}, \ldots\right\}$. Soit $\rho_{\ell_{i}}: \Gamma_{k} \rightarrow \mathbf{Z}_{\ell_{i}}^{\times}$un homomorphisme non ramifié en dehors de $p$ dont l'image est cyclique d'ordre $p^{i}$. La famille des $\rho_{\ell_{i}}$ satisfait à la condition (B) avec $n=1$ et à la condition (ST1) avec $S=\{p\}$; elle ne possède cependant pas la propriété (PR) car son corps d'indépendance est l'unique $\mathbf{Z}_{p}$-extension de $\mathbf{Q}$, qui est de degré infini sur $\mathbf{Q}$.

\footnotetext{
${ }^{2}$ Note ajoutée sur épreuves. Ce problème vient d'être résolu par W. Gadja et S. Petersen : Independence of $\ell$-adic representations over function fields, Compositio Mathematica, à paraître.
} 


\section{§4. Un théorème de finitude sur les corps de nombres}

Soit $d$ un entier $>0$, et soit $G$ un groupe fini. Considérons la condition : $\left(\right.$ Jor $\left._{d}\right)$ Il existe un sous-groupe abélien normal $A$ de $G$ tel que $(G: A) \leqslant d$.

Théorème 2. Pour tout $d>0$ il n'existe qu'un nombre fini de sous-extensions galoisiennes $K / k$ de $\bar{k} / k$ qui sont partout non ramifiées et dont le groupe de Galois a la propriété $\left(\mathrm{Jor}_{d}\right.$ ) ci-dessus.

Démonstration. On sait (Hermite-Minkowski) qu'il n'existe qu'un nombre fini de sous-extensions de $\bar{k}$ de degré $\leqslant d$ qui soient partout non ramifiées (cela provient de ce que leurs discriminants sont bornés en valeur absolue, cf. par exemple [Se 81], $\S 1.4)$. On peut donc trouver une sous-extension finie $k_{1}$ de $\bar{k}$ contenant toutes ces extensions. Soit $k_{2}$ la plus grande extension abélienne non ramifiée de $k_{1}$ contenue dans $\bar{k}$; d'après la théorie du corps de classes, $k_{2}$ est une extension finie de $k_{1}$, donc aussi de $k$. Soit maintenant $K / k$ une extension galoisienne dont le groupe de Galois $G$ a la propriété de l'énoncé, et soit $K^{\prime}$ le sous-corps de $K$ fixé par un sous-groupe abélien normal $A$ d'indice $\leqslant d$. On a $\left[K^{\prime}: k\right] \leqslant(G: A) \leqslant d$ et $K^{\prime}$ est non ramifiée sur $k$. Cela montre que $K^{\prime}$ est contenu dans $k_{1}$. Comme $K / K^{\prime}$ est abélienne et non ramifiée, il en est de même de $K \cdot k_{1} / k_{1}$ et cela entrâne que $K \cdot k_{1}$ est contenu dans $k_{2}$, d'où $K \subset k_{2}$, ce qui prouve la finitude cherchée.

[Ce théorème utilise deux des propriétés les plus importantes des corps de nombres :

a) finitude des extensions de $\mathbf{Q}$ de degré et discriminant bornés ${ }^{3}$;

b) finitude des extensions abéliennes non ramifiées (corps de classes).]

\section{§5. Groupes linéaires d'ordre premier à la caractéristique}

5.1. Le théorème de Jordan classique. Sous sa forme originelle ([Jo 78]), ce théorème s'énonce comme suit :

Théorème 3. Pour tout entier $n \geqslant 0$ il existe un entier $d=d(n)$ tel que tout sousgroupe fini de $\mathbf{G} \mathbf{L}_{n}(\mathbf{C})$ ait la propriété $\left(\mathrm{Jor}_{d}\right) d u \S 4$.

[Autrement dit, un sous-groupe fini de $\mathbf{G L}_{n}(\mathbf{C})$ ne peut être "gros" que s'il contient un gros sous-groupe abélien.]

On trouvera dans [Fr 11] une démonstration simple de ce résultat. Cette démonstration donne une valeur de $d(n)$ telle que

$$
d(n) \leqslant(\sqrt{8 n}+1)^{2 n^{2}} .
$$

On connaît maintenant la valeur optimale de $d(n)$, qui est bien inférieure à celle-là ; ainsi, pour $n \geqslant 71$, on a $d(n)=(n+1)$ !, d'après M. J. Collins [Co 07], améliorant

\footnotetext{
${ }^{3}$ En fait discriminant borné entraîne degré borné, mais cela ne joue aucun rôle ici.
} 
Vol. 88 (2013) Un critère d'indépendance pour une famille de représentations $\ell$-adiques 547

des résultats de B. Weisfeiler et de W. Feit ${ }^{4}$. Nous n'en aurons pas besoin. Dans ce qui suit, nous noterons $d(n)$ n'importe quel entier $d$ pour lequel le théorème 3 est valable.

\subsection{Le théorème de Jordan sur un corps quelconque}

Théorème $\mathbf{3}^{\prime}$. Soient $n$ un entier $\geqslant 0, F$ un corps, $H$ un sous-groupe fini de $\mathbf{G L}_{n}(F)$ et $G$ un quotient de $H$. On suppose que $|G|$ est premier à la caractéristique de $F$ si celle-ci est $\neq 0$. Alors $G$ a la propriété $\left(\operatorname{Jor}_{d(n)}\right) d u \S 4$.

Démonstration. Elle se fait en trois étapes :

5.2.1. Le cas où car $(F)=0$. On peut supposer $F$ de type fini sur $\mathbf{Q}$, donc plongeable dans $\mathbf{C}$. Le théorème 3 montre alors que $H$ a la propriété $\left(\operatorname{Jor}_{d(n)}\right)$ et il en est donc de même de $G$.

5.2.2. Le cas où $\operatorname{car}(F)=p>0$, avec $|H|$ premier à $p$. On peut supposer que $F$ est parfait. Soit $W$ l'anneau des vecteurs de Witt à coefficients dans $F$. On a un homomorphisme surjectif $\mathbf{G L}_{n}(W) \rightarrow \mathbf{G L}_{n}(F)$. Comme $|H|$ est premier à $p, H$ se relève en un sous-groupe de $\mathbf{G L}_{n}(W)$, et l'on applique 5.2.1 au corps des fractions de $W$.

5.2.3. Le cas où $\operatorname{car}(F)=p>0$. Soit $I$ le noyau de $H \rightarrow G$, et soit $P$ un $p$-Sylow de $I$; c'est aussi un $p$-Sylow de $H$, puisque $(H: I)$ est premier à $p$. Soit $N_{H}(P)$ le normalisateur de $I$ dans $H$. On sait (Frattini) que $N_{H}(P) \rightarrow G$ est surjectif ${ }^{5}$. D'autre part, la suite exacte

$$
1 \rightarrow P \rightarrow N_{H}(P) \rightarrow N_{H}(P) / P \rightarrow 1
$$

est scindée car les ordres de $P$ et de $N_{H}(P) / P$ sont premiers entre eux. Il existe donc un sous-groupe $H^{\prime}$ de $N_{H}(P)$, d'ordre premier à $p$, tel que $N_{H}(P)=P . H^{\prime}$. Or l'image de $P$ dans $G$ est triviale, puisque $P$ est contenu dans $I$. On en déduit que $G$ est un quotient de $H^{\prime}$, et l'on conclut en appliquant 5.2.2 à $H^{\prime}$.

\section{§6. Groupes linéaires engendrés par des éléments d'ordre égal à la caractéristique}

Dans ce qui suit, $\ell$ désigne un nombre premier $\geqslant 5$.

6.1. Les groupes simples finis de caractéristique $\ell$ : la famille $\Sigma_{\boldsymbol{\ell}}$. Rappelons comment on définit les groupes simples "du type de Lie" en caractéristique $\ell \geqslant 5$ (pour les propriétés utilisées ici, voir par exemple [GLS 98], §2.2, - noter que l'hypothèse

\footnotetext{
${ }^{4}$ Les démonstrations de Weisfeiler, Feit et Collins dépendent de la classification des groupes finis simples.

${ }^{5}$ L'argument dit "de Frattini" est le suivant: si $h \in H, h P h^{-1}$ est un $p$-Sylow de $I$, donc s'écrit $x P x^{-1}$ avec $x \in I$, d'où $x^{-1} h \in N_{H}(P)$, ce qui montre que $h$ appartient à $I . N_{H}(P)$. On a donc bien $H=I \cdot N_{H}(P)$.
} 
$\ell \geqslant 5$ élimine les cas particuliers exceptionnels que l'on rencontre en caractéristique 2 et 3 , ainsi que les formes tordues à la Suzuki-Ree).

On se donne un groupe algébrique lisse connexe $\underline{H}$ sur un corps fini $F$ dont l'ordre est une puissance de $\ell$. On suppose que $\underline{H}$ est géométriquement simple et simplement connexe, et l'on désigne par $\underline{H}^{\text {adj }}$ le quotient de $\underline{H}$ par son centre. L'image $H_{F}$ de l'homomorphisme $\underline{H}(F) \rightarrow \underline{H}^{\text {adj }}(F)$ est alors un groupe fini simple non abélien.

Remarque. On aurait aussi pu définir $H_{F}$ comme le quotient de $\underline{H}(F)$ par son centre, ou bien comme le sous-groupe de $\underline{H}^{\text {adj }}(F)$ engendré par les $\ell$-Sylow de $\underline{H}^{\text {adj }}(F)$. L'équivalence de ces diverses définitions provient de ce que $\underline{H}(F)$ est engendré par ses éléments unipotents d'après un théorème de Steinberg [St 68], th. 12.4.

Nous noterons $\Sigma_{\ell}$ l'ensemble des classes d'isomorphisme de groupes finis simples qui sont, soit du type $H_{F}$ ci-dessus (pour un $\underline{H}$ et un $F$ convenables $^{6}$ ), soit isomorphe au groupe cyclique $\mathbf{Z} / \ell \mathbf{Z}$.

\subsection{Un lemme}

Lemme 1. Soit $\underline{G}$ un groupe algébrique linéaire connexe sur $\mathbf{F}_{\ell}$ et soit $G=\underline{G}\left(\mathbf{F}_{\ell}\right)$ le groupe de ses points rationnels. Tout quotient simple d'une suite de Jordan-Hölder de $G$ appartient ${ }^{7}$ à $\Sigma_{\ell}$ ou est cyclique d'ordre $\neq \ell$.

Démonstration. Un argument de dévissage permet de supposer que $\underline{G}$ est, soit un groupe unipotent, soit un tore, soit un groupe semi-simple. Les deux premiers cas sont immédiats. On peut donc supposer que $\underline{G}$ est semi-simple. Soit $\underline{\widetilde{G}}$ le revêtement universel de $\underline{G}$ et soit $\underline{G}^{\text {adj }}$ son groupe adjoint. Soient $\widetilde{G}$ et $G^{\text {adj }}$ les groupes de points $\mathbf{F}_{\ell}$-rationnels de ces groupes algébriques. On a des homomorphismes naturels

$$
\widetilde{G} \rightarrow G \rightarrow G^{\text {adj }}
$$

Comme $\underline{\widetilde{G}}$ est simplement connexe, c'est un produit de groupes du type $R_{F / \mathbf{F}_{\ell}} \underline{H}$, où $\underline{H}$ et $F$ sont comme dans 6.1 ci-dessus, et le symbole $R_{F / \mathbf{F}_{\ell}}$ désigne le foncteur "restriction des scalaires" à la Weil (celui que Grothendieck note $\prod_{F / \mathbf{F}_{\ell}}$ ), cf. par exemple [KMRT 98], th. 26.8. On a donc $\widetilde{G}=\prod \underline{H}(F)$. Les homomorphismes

$$
\widetilde{G} \rightarrow G \rightarrow G^{\mathrm{adj}}
$$

ont des noyaux et conoyaux qui sont commutatifs d'ordre premier à $\ell$. De plus, l'image de $\widetilde{G}$ dans $G^{\text {adj }}$ est un produit de groupes simples appartenant à $\Sigma_{\ell}$. Le lemme en résulte.

\footnotetext{
${ }^{6}$ Il y a unicité : un groupe simple n'est isomorphe à $H_{F}$ que pour au plus un couple $(\underline{H}, F)$, à isomorphisme près.

${ }^{7}$ Dans ce qui suit, on dit qu'un groupe simple "appartient” à $\Sigma_{\ell}$ lorsqu'il est isomorphe à un élément de $\Sigma_{\ell}$.
} 
Vol. 88 (2013) Un critère d'indépendance pour une famille de représentations $\ell$-adiques 549

\subsection{Un théorème de Nori}

Théorème 4. Pour tout $n \geqslant 0$, il existe un entier $c(n)$ tel que, si $\ell>c(n)$, tout sous-quotient fini simple de $\mathbf{G L}_{n}\left(\mathbf{Z}_{\ell}\right)$ d'ordre divisible par $\ell$ appartient à $\Sigma_{\ell}$.

Démonstration. Prenons $c(n)=\sup \left(3, c_{2}(n)\right)$, où $c_{2}(n)$ a les propriétés énoncées dans [No 87], Theorem B. Nous allons voir que cet entier convient.

Supposons que $\ell>c(n)$ et soit $H$ un sous-quotient fini simple de $\mathbf{G L}_{n}\left(\mathbf{Z}_{\ell}\right)$ d'ordre divisible par $\ell$. Comme $H$ est simple, cette dernière propriété entraîne que $H$ est engendré par ses $\ell$-Sylow.

L'homomorphisme naturel $\mathbf{G L}_{n}\left(\mathbf{Z}_{\ell}\right) \rightarrow \mathbf{G L}_{n}\left(\mathbf{F}_{\ell}\right)$ est surjectif, et son noyau est un pro- $\ell$-groupe. Il en résulte que $H$ est, soit cyclique d'ordre $\ell$, soit isomorphe à un sous-quotient de $\mathbf{G} \mathbf{L}_{n}\left(\mathbf{F}_{\ell}\right)$. Dans le premier cas, $H$ appartient à $\Sigma_{\ell}$. Dans le second cas, on a $H=G / I$, avec $G \subset \mathbf{G L}_{n}\left(\mathbf{F}_{\ell}\right)$ et $I$ normal dans $G$; on peut évidemment supposer que $G$ est engendré par ses $\ell$-Sylow. D'après [No 87], Theorem B, il existe un $\mathbf{F}_{\ell}$-sous-groupe algébrique connexe $\underline{G}$ de $\mathbf{G} \mathbf{L}_{n}$ tel que $G$ soit contenu dans $\underline{G}\left(\mathbf{F}_{\ell}\right)$ et soit engendré par les $\ell$-Sylow de ce groupe ${ }^{8}$. Le groupe $H$ est un quotient d'une suite de Jordan-Hölder de $G$, donc aussi de $\underline{G}\left(\mathbf{F}_{\ell}\right)$. D'après le lemme 1 , ceci entraîne que $H$ est, soit cyclique d'ordre premier à $\ell$ (ce qui est exclu), soit isomorphe à un élément de $\Sigma_{\ell}$. D'où le théorème.

6.4. Un théorème d'Artin. Le résultat suivant est essentiellement dû à E. Artin ([Ar 55], complété par [KLST 90]) :

Théorème 5. Si $\ell^{\prime}$ est premier $\geqslant 5$ et distinct de $\ell$, on a $\Sigma_{\ell} \cap \Sigma_{\ell^{\prime}}=\varnothing$.

La démonstration donne même un résultat plus fort : si $G$ appartient à $\Sigma_{\ell}$ et $G^{\prime}$ appartient à $\Sigma_{\ell^{\prime}}$, leurs ordres $|G|$ et $\left|G^{\prime}\right|$ sont distincts.

Exemples. Pour $\ell=5$, les ordres des éléments de $\Sigma_{\ell}$, rangés par taille croissante, sont $\{5,60,7800,126000,372000,976500, \ldots\}$.

Pour $\ell=7$, ce sont $\{7,168,58800,1876896,5663616,20176632, \ldots\}$.

\section{§7. Deux critères d'indépendance}

7.1. Un critère élémentaire. Revenons aux notations du $\S 1$, et soit $\rho_{i}: \Gamma \rightarrow G_{i}$, $i \in I$, une famille d'homomorphismes, les groupes $\Gamma$ et $G_{i}$ étant des groupes profinis, et les $\rho_{i}$ étant continus.

Lemme 2. Supposons que les groupes $\rho_{i}(\Gamma) \subset G_{i}$ aient la propriété suivante :

(D) Si $i \neq j$, aucun quotient fini simple de $\rho_{i}(\Gamma)$ n'est isomorphe à un quotient de $\rho_{j}(\Gamma)$.

\footnotetext{
${ }^{8}$ La définition de $\underline{G}$ donnée par Nori est très simple : c'est le plus petit sous-groupe algébrique de $\mathbf{G L}_{n}$ contenant les groupes à 1 paramètre $t \mapsto u^{t}$, où $u$ parcourt les éléments d'ordre $\ell$ de $G$. Dans la terminologie de [Se 94], §4, c'est le saturé de $G$.
} 
Alors les $\rho_{i}$ sont indépendants.

Démonstration. On peut évidemment supposer que les $\rho_{i}$ sont surjectifs, i.e. $G_{i}=$ $\rho_{i}(\Gamma)$ pour tout $i$.

Considérons d'abord le cas où $I$ est un ensemble à deux éléments, par exemple $I=\{1,2\}$. Si $\rho: \Gamma \rightarrow G_{1} \times G_{2}$ n'est pas surjectif, le classique lemme de Goursat montre qu'il existe un groupe profini non trivial $A$ et des homomorphismes surjectifs $f_{i}: G_{i} \rightarrow A$ tels que $f_{1} \circ \rho_{1}=f_{2} \circ \rho_{2}$. Comme $A$ est non trivial, il a un quotient qui est un groupe simple fini, et ce groupe est quotient à la fois de $G_{1}$ et de $G_{2}$, contrairement à l'hypothèse (D).

Le cas où $I$ est fini se déduit par récurrence sur $|I|$ du cas où $|I|=2$, et le cas où $|I|$ est infini se déduit par passage à la limite du cas où $|I|$ est fini.

7.2. Un autre critère. Soit $\Gamma$ un groupe profini et soit $L$ un ensemble de nombres premiers. Pour tout $\ell \in L$, soit $\rho_{\ell}: \Gamma \rightarrow G_{\ell}$ un homomorphisme continu de $\Gamma$ dans un groupe de Lie $\ell$-adique compact $G_{\ell}$.

Lemme 3. Supposons qu'il existe une partie finie I de L telle que la famille $\left(\rho_{\ell}\right)_{\ell \in L-I}$ ait la propriété $(\mathrm{PR}) d u$ §1. Alors il en est de même de la famille $\left(\rho_{\ell}\right)_{\ell \in L}$.

[Autrement dit, pour prouver (PR), on a le droit de supprimer un nombre fini d'éléments de $L$.]

Démonstration. On peut supposer que $I$ est réduit à un seul élément, que l'on notera $p:$ le cas général en résultera par récurrence sur $|I|$. Quitte à remplacer $\Gamma$ par un sous-groupe ouvert, on peut supposer que les $\rho_{\ell}$ sont indépendants pour $\ell \neq p$; on peut aussi supposer que tous les $\rho_{\ell}$ sont surjectifs. Nous allons alors démontrer un peu mieux que (PR), à savoir :

(*) La famille des $\rho_{\ell}$ possède la propriété (RO) $d u$ §1.

Autrement dit, l'image de $\Gamma$ par l'homomorphisme

$$
\rho=\left(\rho_{\ell}\right): \Gamma \rightarrow G_{p} \times \prod_{\ell \neq p} G_{\ell}
$$

est ouverte dans $\prod_{\ell} G_{\ell}$.

Les deux projections $\rho(\Gamma) \rightarrow G_{p}$ et $\rho(\Gamma) \rightarrow \prod_{\ell \neq p} G_{\ell}$ sont surjectives par hypothèse. On se trouve donc dans la situation du lemme de Goursat. Autrement dit, si l'on identifie $G_{p}$ au facteur $G_{p} \times 1$ de $G_{p} \times \prod_{\ell \neq p} G_{\ell}$, le groupe quotient $C=G_{p} /\left(\rho(\Gamma) \cap G_{p}\right)$ est un quotient de $\prod_{\ell \neq p} G_{\ell}$. Dire que $\rho(\Gamma)$ est ouvert équivaut à dire que $C$ est fini. C'est ce que nous allons démontrer.

Observons d'abord que $C$ est un groupe de Lie $p$-adique compact (puisque c'est un quotient de $G_{p}$ ); il contient donc un sous-groupe ouvert normal $U$ qui est un pro$p$-groupe sans torsion (cf. par exemple [Se 65], II, §IV.9, th. 5, [Bo 72], Chap. III, $\S 7$, ou [DSMS 99], th. 8.32). Si $J$ est une partie finie de $L-\{p\}$, notons $C_{J}$ l'image de l'homomorphisme

$$
\prod_{\ell \in J} G_{\ell} \rightarrow \prod_{\ell \neq p} G_{\ell} \rightarrow C
$$


Vol. 88 (2013) Un critère d'indépendance pour une famille de représentations $\ell$-adiques 551

Les $p$-Sylow des $G_{\ell}$ sont finis si $\ell \in J$; il en est donc de même de ceux de $C_{J}$. Comme $U$ est sans torsion, cela montre que $U \cap C_{J}=1$; d'où $\left|C_{J}\right| \leqslant(C: U)$. Cela donne une borne uniforme pour l'ordre de $C_{J}$, ce qui entraîne qu'il existe un $C_{J}$ qui contient tous les autres. Mais la réunion des $C_{J}$ est dense dans $C$. D'où le fait que $C$ est fini.

\section{§8. Démonstration du théorème 1}

Revenons à la situation du théorème 1 , relative à un homomorphisme

$$
\rho=\left(\rho_{\ell}\right): \Gamma_{k} \rightarrow \prod_{\ell \in L} G_{\ell}
$$

satisfaisant aux conditions (B) et (PST). Pour prouver que $\rho$ a la propriété (PR), nous procéderons en plusieurs étapes.

8.1. Réductions. Quitte à remplacer $k$ par une extension finie, on peut supposer que la condition de semi-stabilité (ST) est satisfaite. On peut aussi supposer que les $\rho_{\ell}$ sont surjectifs. D'après (B), on peut choisir un entier $n \geqslant 0$ tel que, pour tout $\ell \in L$, le groupe $G_{\ell}$ soit un sous-quotient de $\mathbf{G} \mathbf{L}_{n}\left(\mathbf{Z}_{\ell}\right)$. D'après le lemme 3 , on peut aussi supposer que tous les $\ell \in L$ sont $>\sup (3, c(n))$ où $c(n)$ a la propriété énoncée dans le théorème 4 . Pour la même raison, on peut aussi supposer que l'on a $\ell \neq p_{v}$ pour toute place $v$ de l'ensemble fini $S$ intervenant dans (ST).

8.2. Les groupes $\boldsymbol{A}_{\ell}$. Si $\ell \in L$, notons $\Gamma_{k, \ell}$ le plus petit sous-groupe normal fermé de $\Gamma_{k}$ contenant les groupes d'inertie $I_{\bar{v}}$ correspondant aux places $v$ telles que $p_{v}=\ell$. D'après (ST1), on a $\rho_{\ell^{\prime}}\left(\Gamma_{k, \ell}\right)=1$ pour tout $\ell^{\prime} \neq \ell$. L'image du groupe $\Gamma_{k, \ell}$ par $\rho: \Gamma_{k} \rightarrow \prod G_{\ell}$ est donc contenue dans le $\ell$-ième facteur de $\prod G_{\ell}$. Notons $A_{\ell}$ cette image; c'est un sous-groupe fermé normal de $G_{\ell}$. Le plus petit sous-groupe fermé de $\prod G_{\ell}$ contenant tous les $A_{\ell}$ n'est autre que le produit $\prod A_{\ell}$. En particulier, on a :

Lemme 4. Le sous-groupe $\rho\left(\Gamma_{k}\right)$ de $\prod G_{\ell}$ contient $\prod A_{\ell}$.

8.3. Les groupes $G_{\ell}^{+}$. Si $\ell \in L$, notons $G_{\ell}^{+}$le sous-groupe de $G_{\ell}$ engendré par ses $\ell$-Sylow; c'est un sous-groupe ouvert normal de $G_{\ell}$. Posons $H_{\ell}=G_{l} / G_{\ell}^{+}$. $A_{\ell}$; c'est un groupe fini d'ordre premier à $\ell$.

Lemme 5. a) L'homomorphisme $\Gamma_{k} \rightarrow G_{\ell} \rightarrow H_{\ell}$ est partout non ramifié.

b) Le groupe $H_{\ell}$ jouit de la propriété $\operatorname{Jor}_{d(n)}$ des $\$ \S 4-5$.

Démonstration. Soit $v \in V_{k}$, et soit $\bar{v}$ une place de $\bar{k}$ prolongeant $v$. Si $p_{v}=\ell$, on a $\rho_{\ell}\left(I_{\bar{v}}\right) \subset A_{\ell}$ par définition de $A_{\ell}$; l'image de $I_{\bar{v}}$ dans $H_{\ell}$ est donc triviale. Si $p_{v} \neq \ell$, le groupe $\rho_{\ell}\left(I_{\bar{v}}\right)$ est un pro- $\ell$-groupe d'après $(\mathrm{ST})$; il est donc contenu dans $G_{\ell}^{+}$et son image dans $H_{\ell}$ est triviale. Cela démontre a).

Quant à b), il résulte du fait que l'ordre de $H_{\ell}$ est premier à $\ell$, ce qui permet de lui appliquer le théorème $3^{\prime}$. 
8.4. Changement de corps. D' après le lemme 5 , les homomorphismes $\Gamma_{k} \rightarrow H_{\ell}$ sont non ramifiés. Comme les $H_{\ell}$ ont la propriété $\mathrm{Jor}_{d(n)}$, on peut appliquer le théorème 2 . On en déduit qu'il existe une extension finie non ramifiée $k^{\prime}$ de $k$ telle que, pour tout $\ell \in L$, l'image de $\rho_{\ell}\left(\Gamma_{k^{\prime}}\right)$ dans $H_{\ell}$ soit triviale. Choisissons une telle extension. On a alors $\rho_{l}\left(\Gamma_{k^{\prime}}\right) \subset G_{\ell}^{+} . A_{\ell}$ pour tout $\ell$. Nous allons maintenant prendre $k^{\prime}$ comme corps de base ; nous poserons $G_{\ell}^{\prime}=\rho_{\ell}\left(\Gamma_{k^{\prime}}\right)$, et nous noterons $G_{\ell}^{\prime+}$ et $A_{\ell}^{\prime}$ les groupes correspondant à $G_{\ell}^{+}$et à $A_{\ell}$; par exemple, $G_{\ell}^{\prime+}$ est le sous-groupe de $G_{\ell}^{\prime}$ engendré par les $\ell$-Sylow de $G_{\ell}^{\prime}$.

Lemme 6. Si $\ell>\left[k^{\prime}: k\right]$, on a $G_{\ell}^{\prime+}=G_{\ell}^{+}, A_{\ell}^{\prime}=A_{\ell}$ et $G_{\ell}^{\prime}=G_{\ell}^{\prime+} \cdot A_{\ell}^{\prime}$.

Démonstration. L'hypothèse faite sur $\ell$ entraîne que l'indice de $G_{\ell}^{\prime}$ dans $G_{\ell}$ est $<\ell$, d'où le fait que tout $\ell$-Sylow de $G_{\ell}$ est contenu dans $G_{\ell}^{\prime}$, ce qui entraîne $G_{\ell}^{\prime+}=G_{\ell}^{+}$. L'égalité $A_{\ell}^{\prime}=A_{\ell}$ résulte de ce que les groupes d'inertie $I_{\bar{v}}$ sont les mêmes pour $k^{\prime}$ et pour $k$, puisque $k^{\prime}$ est non ramifié sur $k$. Enfin, l'égalité $G_{\ell}^{\prime}=G_{\ell}^{\prime+} \cdot A_{\ell}^{\prime}$ résulte de ce que $G_{\ell}^{\prime}=\rho_{\ell}\left(\Gamma_{k^{\prime}}\right)$ est contenu dans $G_{\ell}^{+} \cdot A_{\ell}$.

8.5. Fin de la démonstration. D'après le lemme 3 , on peut supposer que l'on a $\ell>\left[k^{\prime}: k\right]$ pour tout $\ell \in L$. Le lemme 6 montre que l'on a alors $G_{\ell}^{\prime}=G_{\ell}^{\prime+} \cdot A_{\ell}^{\prime}$ pour tout $\ell$. D'après le théorème 4 , tout quotient simple de $G_{\ell}^{\prime+}$ appartient à l'ensemble $\Sigma_{\ell}$ défini au n ${ }^{0} 6.1$. Il en est donc de même des quotients simples de $G_{\ell}^{\prime} / A_{\ell}^{\prime}$. Comme les $\Sigma_{\ell}$ sont deux à deux disjoints (théorème 5), on peut appliquer le lemme 2 à la famille des homomorphismes $\Gamma_{k^{\prime}} \rightarrow G_{\ell}^{\prime} / A_{\ell}^{\prime}$. On en conclut que l'homomorphisme $\Gamma_{k^{\prime}} \rightarrow \prod G_{\ell}^{\prime} / A_{\ell}^{\prime}$ est surjectif. Si l'on pose $X^{\prime}=\rho\left(\Gamma_{k^{\prime}}\right)$ et $A^{\prime}=\prod A_{\ell}^{\prime}$, cela revient à dire que $X^{\prime} . A^{\prime}=\prod G_{\ell}^{\prime}$. Mais le lemme 4, appliqué au corps $k^{\prime}$, montre que $X^{\prime}$ contient $A^{\prime}$. On a donc $X^{\prime}=\prod G_{\ell}^{\prime}$, ce qui achève la démonstration.

\section{Références}

[Ar 55] E. Artin, The orders of the classical simple groups. Comm. Pure Appl. Math. 8 (1955), 455-472 (= C.P., $\mathrm{n}^{\circ}$ 33). Zbl 0065.25703 MR 0073601

[Be 96] P. Berthelot, Altération des variétés algébriques (d'après A. J. de Jong). Dans Sém. Bourbaki 1995/96, exposé 815 ; Astérisque 241 (1997), Soc. Math. France, Paris 1997, 273-311. Zbl 0924.14007 MR 1472543

[BLR 90] S. Bosch, W. Lütkebohmert and M. Raynaud, Néron models. Ergeb. Math. Grenzgeb. (3) 21, Springer-Verlag, Berlin 1990. Zbl 0705.14001 MR 1045822

[Bo 72] N. Bourbaki, Groupes et Algèbres de Lie. Chapitre II : Algèbres de Lie libres. Chapitre III : Groupes de Lie. Actualités Scientifiques et Industrielles 1349, Hermann, Paris 1972. Zbl 0244.22007 MR 0573068

[Bo 00] E. Bouscaren, Théorie des modèles et conjecture de Manin-Mumford (d'après Ehud Hrushovski). Dans Sém. Bourbaki 1999/2000, exposé 870; Astérisque 276 (2002), Soc. Math. France, Paris 2002, 137-159. Zbl 0994.03033 MR 1886759 
Vol. 88 (2013) Un critère d'indépendance pour une famille de représentations $\ell$-adiques 553

[Co 07] M. J. Collins, On Jordan's theorem for complex linear groups. J. Group Theory 10 (2007), 411-423. Zbl 1125.20033 MR 2334748

[DSMS 99] J. D. Dixon, M. P. F. du Sautoy, A. Mann, and D. Segal, Analytic pro-p-groups. Second edition, revised and enlarged by Marcus du Sautoy and Dan Segal, Cambridge Stud. Adv. Math. 61, Cambridge University Press, Cambridge 1999. Zbl 0934.20001 MR 1720368

[Fr 11] F. G. Frobenius, Über den von L. Bieberbach gefundenen Beweis eines Satzes von C. Jordan. Sitz. Königlich Preuss. Akad. Wiss. Berlin (1911), 241-248 (= Ges. Abh. III, 493-500). JFM 42.0152.01

[GLS 98] D. Gorenstein, R. Lyons and R. Solomon, The classification of the finite simple groups. Number 3, Part I, Chapter A, Almost simple K -groups, Math. Surveys Monogr. 40.3, Amer. Math. Soc., Providence, RI, 1998 Zbl 0890.20012 MR 1490581

[Il 10] L. Illusie, Constructibilité générique et uniformité en $\ell$. Orsay, 2010, non publié.

[Jo 96] A. J. de Jong, Smoothness, semi-stability and alterations. Inst. Hautes Études Sci. Publ. Math. 83 (1996), 51-93. Zbl 0916.14005 MR 1423020

[Jo 78] C. Jordan, Mémoire sur les équations différentielles linéaires à intégrale algébrique. Borchardt J. (J. reine angew. Math.) 84 (1878), 89-215 (= Oe.II, 13-140). JFM 09.0234.02

[KL 86] N. M. Katz et G. Laumon, Transformation de Fourier et majoration de sommes exponentielles. Inst. Hautes Études Sci. Publ. Math. 62 (1986), 361-418; Erratum, Publ. Math. IHES 69 (1989), 233. Zbl 0603.14015 MR 0823177

[KLST 90] W. Kimmerle, R. Lyons, R. Sandling, and D. N. Teague, Composition factors from the group ring and Artin's theorem on orders of simple groups. Proc. London Math. Soc. 60 (1990), 89-122. Zbl 0668.20009 MR 1023806

[KMRT 98] M-A. Knus, A. Merkurjev, M. Rost, and J-P. Tignol, The book of involutions. Amer. Math. Soc. Colloq. Publ. 44, Amer. Math. Soc., Providence, RI, 1998. Zbl 0955.16001 MR 1632779

[No 87] M. V. Nori, On subgroups of $\mathbf{G L}_{n}\left(\mathbf{F}_{p}\right)$. Invent. math. 88 (1987), 257-275. Zbl 0632.20030 MR 0880952

[Se 65] J-P. Serre, Lie algebras and Lie groups. Benjamin Publ., New York 1965 ; Lecture Notes in Math. 1500, Springer-Verlag, Berlin 1992 ; corrected fifth printing, 2006. Zbl 0132.27803 MR 0218496

[Se 81] J-P. Serre, Quelques applications du théorème de densité de Chebotarev. Inst. Hautes Études Sci. Publ. Math. 54 (1981), 123-201 (= Oe.III, n $\left.{ }^{\circ} 125\right)$. Zbl 0496.12011 MR 0644559

[Se 86] J-P. Serre, Lettre à Ken Ribet du 7/3/1986 (= Oe.IV, ${ }^{\circ}$ 138). Zbl 0933.01034 MR 1730973

[Se 91] J-P. Serre, Propriétés conjecturales des groupes de Galois motiviques et des représentations $\ell$-adiques. Dans Motives, Proc. Symp. Pure Math. 55, Part 1, Amer. Math. Soc., Providence, RI, 1994, 377-400 (= Oe.IV, n 161). Zbl 0812.14002 MR 1265537

[Se 94] J-P. Serre, Sur la semi-simplicité des produits tensoriels de représentations de groupes. Invent. math. 116 (1994), 513-530 (= Oe.IV, $\mathrm{n}^{\circ}$ 164). Zbl 0816.20014 MR 1253203 
[SGA 4] M. Artin, A. Grothendieck et J-L. Verdier, Théorie des topos et cohomologie étale des schémas. 3 vol., Lecture Notes in Math. 269, 270, 305, Springer-Verlag, Berlin 1972-1973. Zbl 0234.00007, Zbl 0237.00012, Zbl 0245.00002, MR 0354652, MR 0354653, MR 0354654

[SGA 7 I] A. Grothendieck, Groupes de monodromie en géométrie algébrique. Lecture Notes in Math. 288, Springer-Verlag, Berlin 1972. Zbl 0237.00013 MR 0354656

[St 68] R. Steinberg, Endomorphisms of linear algebraic groups. Mem. Amer. Math. Soc. 80, Amer. Math. Soc., Providence, R.I., 1968 (= C.P., n ${ }^{\circ}$ 23). Zbl 0164.02902 MR 0230728

Received May 24, 2010

Jean-Pierre Serre, Collège de France, 3 rue d'Ulm, 75005 Paris, France

E-mail: serre@noos.fr 